\title{
Evolution of a novel and adaptive floral scent in wild tobacco
}

Han Guo ${ }^{1}$, Nathalie D. Lackus², Tobias G. Köllner ${ }^{2}$, Ran Li ${ }^{1}$, Julia Bing ${ }^{1}$, Yangzi Wang ${ }^{3}$, Ian T. Baldwin ${ }^{1}$ and Shuqing $\mathrm{Xu}^{3 *}$

1, Department of Molecular Ecology, Max Planck Institute for Chemical Ecology, HansKnöll-Strasse 8, DE-07745 Jena, Germany

2 Department of Biochemistry, Max Planck Institute for Chemical Ecology, Hans-KnöllStrasse 8, DE-07745 Jena, Germany

3, Institute for Evolution and Biodiversity, University of Münster, Hüfferstrasse 1, DE-48149

Münster, Germany

*Correspondence: shuqing.xu@uni-muenster.de 


\section{Abstract}

Many plants emit diverse floral scents that mediate plant-environment interactions and attain reproductive success. However, how plants evolve novel adaptive floral volatiles remains unclear. Here, we show that in the wild tobacco, Nicotiana attenuata, a dominant

5 species-specific floral volatile (benzyl acetone, BA) that attracts pollinators and deters

6 florivore is synthesized by phenylalanine ammonia-lyase 4 (NaPAL4), isoflavone reductase 3

7 (NaIFR3), and chalcone synthase 3 (NaCHAL3). Transient expression of NaFIR3 alone in $N$.

8 attenuata leaves is sufficient and necessary for ectopic foliar BA emissions, and the BA

9 emission level is increased by co-expressing NaIFR 3 with NaPAL4 and NaCHAL3.

10 Independent changes in transcription in all three genes contributed to intraspecific variations of floral BA emission. However, among species, the gain-of-expression in NaIFR3 resulted in the biosynthesis of BA that was only found in N. attenuata. This study suggests that novel metabolic pathways associated with adaptation can arise via re-configurations of gene expression. 


\section{Introduction}

One of the major challenges in evolutionary biology is to understand the genetic mechanisms underlying the origin of phenotypic novelties. In flowering plants, floral volatiles are highly diverse and important for mediating ecological interactions between flowers and their visitors, including pollinators, florivores and pathogens ${ }^{1,2}$. In contrast to ubiquitous floral volatiles that are involved in the full spectrum of plant-pollinator interactions among different plant species ${ }^{3}$, species-specific floral volatiles likely evolved as the consequence of local adaption ${ }^{4,5}$. Although many of these species-specific floral volatiles are considered as novel adaptive traits in plants, how did they evolve remains largely unclear.

Benzyl acetone (4-phenylbutan-2-one; BA), a dominant nocturnal floral volatile in the wild tobacco species Nicotiana attenuata ${ }^{6,7}$, is known to attract hawkmoth pollinators such as Manduca sexta for outcrossing ${ }^{8-10}$ and simultaneously deter feedings from the florivore Diabrotica undecimpunctata ${ }^{11}$. Intriguingly, BA was not found in other Nicotiana species $^{12}$, suggesting that BA is a species-specific floral volatile that underwent rapid evolution. Previous studies have shown that a chalcone synthase ( $N a C H A L 3)$ is involved in the BA biosynthesis, as silencing this $N a C H A L 3$ resulted in reduced BA emissions in N. attenuata $^{8-10}$. However, the biosynthetic machinery of BA and its evolution remain a mystery.

To identify genes involved in floral BA biosynthesis, we conducted QTL mapping, gene co-expression network analysis and genetic manipulations. We demonstrated that floral BA is synthesized from L-phenylalanine via three enzymes: phenylalanine ammonia-lyase 4 (NaPAL4), isoflavone reductase 3 (NaIFR3), and NaCHAL3. Comparative and evolutionary analyses among closely related species further suggest that the species-specific floral BA emission is resulted from a recent gain-of-expression in corolla limb in NaIFR3, a gene that originated before the divergence of Nicotiana. This study provides an example that novel metabolic pathways can arise via re-configuration the expression of existing genes.

\section{Results and discussion}

\section{NaPAL4 is required for the biosynthesis of BA in $N$. attenuata flowers.}

To identify the genetic basis underlying the variation in floral BA emissions, we performed quantitative trait loci (QTL) mapping using a $N$. attenuata advanced intercross recombinant inbred line (AI-RIL) population ${ }^{13}$, which was developed by crossing two inbred lines (AZ and UT) that differ in floral BA emissions $(p=0.0047$, Figure $1 A)$. By measuring the floral BA emission among individuals in the AI-RIL population, we identified one QTL locus on linkage group 5 (Figure 1B) that is significantly associated with floral BA emission. Because the genome of $N$. attenuata remains fragmented, to identify the candidate genes, we compared the corresponding genomic information of the identified QTL in N. attenuata and their syntenic genome annotations in Petunia. We found two homologous of phenylalanine ammonia-lyase (PAL) genes located at the corresponding QTL region. The PAL enzyme is involved in converting L-phenylalanine to trans-cinnamic acid $(t-\mathrm{CA})$, which is the first step of most benzenoid metabolisms in Petunia ${ }^{14}$.

To further examine whether BA is synthesized from L-phenylalanine, we stem-fed $N$. attenuata inflorescences (UT genotype) with deuterium-labeled phenylalanine (L-phenyl- $\mathrm{d}_{5}$ alanine) and measured floral BA emissions. In comparison to controls (water), the plants fed by L-phenyl- $\mathrm{d}_{5}$-alanine emitted significantly more BA $(\mathrm{p}=0.007$, Figure $1 \mathrm{C})$. Furthermore, 
mass-spectrum analysis revealed that inflorescences feeding with L-phenyl- $\mathrm{d}_{5}$-alanine resulted in the occurrence of deuterium-labeled BA (Figure 1D), suggesting BA is synthesized from L-phenylalanine.

In the N. attenuata genome, we found four NaPAL candidates (NaPAL1-4). To examine the enzymatic activities of these NaPALs in vitro, we heterologously expressed NaPAL1-4 in Escherichia coli. The results showed that all four NaPALs converted the substrate Lphenylalanine into $t$-CA in vitro (Figures S1A-S1F). We then compared the transcript abundance of these four candidates in the corolla limb, the tissue that is responsible for the emission of $\mathrm{BA}^{6}$, between the two parental lines that were used to generate the AI-RIL (UT and AZ). While the transcript abundance of NaPAL1/2/3 in the corolla limb is similar between AZ and UT (Figures S1G-S1I), NaPAL4 was only transcribed in UT but not in AZ (p $<0.001$, Figure 1E). Further southern blot analysis showed that NaPAL4 was absent in the AZ genome (Figures S1J and S1K).

To determine the function of NaPAL4 in vivo, we specifically silenced the expression of each NaPAL in N. attenuata UT plants using virus-induced gene silencing (VIGS) (Figure S1L) and measured their floral BA emission, respectively. Although NaPAL1/2/3/4 could all convert L-phenylalanine into $t$-CA in vitro, only NaPAL4-silenced plants showed reduced floral BA emissions ( $\mathrm{p}<0.001$, Figures $1 \mathrm{~F}$ and $\mathrm{S} 1 \mathrm{M})$. Moreover, kinetic gene expression analysis of NaPAL1-4 in eight different organs showed that NaPAL4 had the highest transcript abundance in the corolla limb at $\sim$ 20:00 (Figures S1N-S1Q), which is consistent to its role in BA biosynthesis. Additional analysis on the subcellular localization of NaPAL4 showed that NaPAL4 is localized to the endoplasmic reticulum membrane (Figures S1R-S1U), which is similar to many other phenylpropanoid biosynthesis-related PALs ${ }^{15}$. Taken together, these results suggest that the BA biosynthesis in N. attenuata requires NaPAL4-mediated conversion of L-phenylalanine to $t$-CA.

\section{NaIFR3 is co-transcribed with $\mathrm{NaPAL4}, \mathrm{NaCHAL3}$ and necessary for BA biosynthesis.}

Because $t$-CA has an extra carbon-carbon double bond in comparison to BA, we hypothesized that a reductase that removes the double bond is involved in the BA biosynthesis in N. attenuata (Figure 2A). To test this hypothesis, we first searched for the genes that are co-expressed with NaPAL4 using our previously established Nicotiana attenuata datahub platform ${ }^{16}$. In addition, since previous study showed that a chalcone synthase, $N a C H A L 3$ (renamed from NaCHAL1 to NaCHAL3 according to phylogenetic analysis in this study), is involved in the BA biosynthesis ${ }^{8}$, we also included $\mathrm{NaCHAL3}$ in the gene co-expression analysis. Among all co-expressed genes, NaIFR3, which structurally belongs to the family of NADPH-dependent reductases ${ }^{17}$, showed a similar corolla limb-specific expression to both NaPAL4 and NaCHAL3 (Figure 2B). A kinetic of the transcript abundance showed that NaPAL4, NaIFR3 and NaCHAL3 are all highly transcribed in the night (Figure 2C), which is consistent to the nocturnal floral emission of $\mathrm{BA}^{6,11}$.

To examine the function of NaIFR3 in vivo, we specifically silenced the expression of NaIFR3 in N. attenuata (UT) using VIGS ( $<<0.001$, Figures 2D top panel and S2A) and measured the floral BA emission. The silencing of NaIFR 3 did not result in any morphological changes of the flowers, but specifically reduced the floral BA emission in $N$. attenuata $(\mathrm{p}<0.001$, Figure 2D bottom panel). 
We then compared the transcript abundance of NaIFR 3 between AZ and UT in corolla limbs. Interestingly, while NaIFR3 was highly transcribed in UT, it is only transcribed at the basal level in AZ ( $<<0.001$, Figure S2B). Further southern blot analysis showed that the low transcript abundance of NaIFR3 in AZ is not because of a gene loss (Figure S2C). Because only one locus was found in the QTL mapping, it seems likely that NaPAL4 and the cis- or trans- regulator that resulted in differences in the transcript abundance of NaIFR3 are colocated.

Due to the difficulties to obtain a stable substrate, it is challenging to directly examine the biochemical function of NaIFR 3 in vitro. Therefore, we directly examined the function of NaIFR3 by ectopically expressing NaIFR3, NaPAL4 and NaCHAL3 in N. attenuata leaves, either individually or in combinations. At 48 hours after transformation, ectopic transcript abundance of NaPAL4, NaIFR3 and NaCHAL3 and foliar BA emission were measured in the transformed $N$. attenuata rosette leaves. The results showed that ectopic expression of NaIFR 3 alone is already sufficient for low-levels of foliar BA emission (Figure 3A), suggesting that other components of BA biosynthesis already exist in N. attenuata leaves. Consistently, in $N$. attenuata leaves, we found a relatively high transcript abundance of NaPAL1 and 2 (Figures $\mathrm{S} 1 \mathrm{~N}$ and $\mathrm{S} 1 \mathrm{O}$ ) both showing in vitro the ability to convert Lphenylalanine into $t$-CA (Figures S1C and S1D) and we observed transcripts of NaCHAL3 (Figures 2B and S2K), albeit in low abundance. Further co-expression of NaIFR3 with NaPAL4 and NaCHAL3 in N. attenuata leaves, either in pairwise combinations or all three together, significantly increased BA emission in comparison to expressing NaIFR 3 alone (Figure 3A). These results revealed that NaIFR3 is required for BA biosynthesis in $N$. attenuata and co-expression of NaPAL4, NaIFR 3 and NaCHAL3 in leaves is sufficient for ectopic foliar BA emission.

\section{Independent expression changes of $\mathrm{NaPAL4}, \mathrm{NaIFR} 3$ and $\mathrm{NaCHAL3}$ resulted in intraspecific variations of floral BA emission.}

To further investigate the genetic mechanisms underlying natural variations of floral BA emission in N. attenuata, we measured the transcript abundance of NaPAL4, NaIFR 3 and NaCHAL3 3 and the floral BA emission among 22 natural accessions (Table S3) that had been re-sequenced with low coverage. Overall, the variations in floral BA emission and the transcript abundance of NaPAL4, NaIFR 3 and NaCHAL3 did not show a clear correlation with their genetic distance that was calculated using genome-wide SNPs (Figure 3B). This suggests that the variations of floral BA emission were not a result from historic demographic changes of $N$. attenuata, but likely due to variations of local adaptations to pollinators or florivores ${ }^{18}$. Furthermore, expression changes in NaPAL4, NaIFR 3 and NaCHAL3 were also not correlated among genotypes, indicating that changes in the expression among these genes were largely independent.

We then estimated the extent to which the expression changes of each of the three genes contributed to the natural variation of floral BA emissions in $N$. attenuata. The results showed that the changes in the transcript abundance of NaPAL4, NaIFR3, NaCHAL3 and all three genes together could explain $\sim 38 \%, \sim 50 \%, \sim 70 \%$ and $\sim 85 \%$ of the floral BA emission variance among the 22 accessions, respectively (Figure 3C). In contrast, variations in NaPAL1, 2, 3 and 1-3 together, which are likely not involved directly in the floral BA 
biosynthesis, can only explain $\sim 11 \%, \sim 5 \%, \sim 10 \%$ and $\sim 15 \%$ of the floral BA emissions, respectively (Figure S2E). Together, these results suggest that the intraspecific variations in the floral BA emission in $N$. attenuata largely resulted from independent changes in the expression of each of its biosynthetic genes.

\section{Gain-of-expression in NaIFR3 resulted in the BA biosynthesis in N. attenuata.}

Based on both the in vivo functions and the putative enzymatic activities of NaPAL4, NaIFR3 and NaCHAL3, we derived a possible BA biosynthesis pathway in N. attenuata (Figure S3A). It is also possible that NaCHAL3 might act earlier than NaIFR3 in the pathway. However, a previous study in Rheum palmatum showing that the benzalacetone synthase (BAS), which shares $70 \%$ amino acid sequence similarity to $\mathrm{NaCHAL3}$, catalyzes the onestep decarboxylative condensation of 4-coumaroyl-CoA with malonyl-CoA to produce a diketide benzalacetone ${ }^{19,20}$. Therefore, it is likely that NaCHAL3 is the enzyme that is responsible for the final product of BA emission.

To investigate the evolution of floral BA biosynthesis in $N$. attenuata, we first performed phylogenomic analysis using the available genomic data and analyzed the evolutionary history of NaPAL4, NaIFR3 and NaCHAL3. The results showed that NaPAL4 originated before the whole genome triplication (WGT) that was shared among Solanaceae species (Figures 4A and S3B), while NaIFR3 and NaCHAL3 originated from gene duplications that are specific to the Nicotiana genus (Figures 4A, S3C and S3D). The duplicated copies of NaPAL, NaIFR and NaCHAL all showed expression divergence, both among tissues and in their temporal dynamics (Figures S1N-S1Q and S2F-S2K). Together, these results suggest that the BA biosynthesis resulted from recruiting both ancient and recent duplicated genes.

Because $N$. sylvestris is the most closely related species to $N$. attenuata that has its genomic sequence available, phylogenomic analysis is limited to the time before speciation between $N$. sylvestris and $N$. attenuata (Figure 4A). To gain more insights into the evolution of BA biosynthesis after this speciation event, we further analyzed the floral volatiles in seven closely related Nicotiana species (Figures 4B and 4C). Consistent with a previous study ${ }^{12}$, BA was only found in N. attenuata (Figure 4C).

We amplified and sequenced the cDNA of IFR and CHAL among the seven Nicotiana species. Phylogenetic analyzes of the cDNA sequences of IFR and CHAL among these closely related species were consistent to the analysis based on the genomic sequences: NaIFR3 originated in the ancestor of $N$. attenuata and $N$. obtusifolia $(\sim 12.5 \mathrm{MYA})^{21}$ and NaCHAL3 occurs specifically in the clade of Petunioides ( 9.1 MYA $)^{22}$.

We further analyzed the expression kinetics of NaPAL4, NaIFR3 and NaCHAL3 in the corolla limb of all seven species. Interestingly, like in N. attenuata, NaPAL4 showed a high transcript abundance at 20:00 among all species (Figure 4D) and NaCHAL3 was highly transcribed at 20:00 among the four species of Petunioides. This indicates that NaPAL4 and NaCHAL3 might be involved in other floral metabolic pathways in the different Nicotiana species. However, although NaIFR3 existed in all four species of Petunioides, it was only transcribed in N. attenuata corolla limbs and showed evening-specific expression pattern. Because NaIFR 1/2, the ancestor/homologs of NaIFR3, were expressed either in styles, stems or leaves, the corolla limb expression of NaIFR3 is likely due to a tissue-specific gain-ofexpression event. The event either specifically occurred in N. attenuata or in the ancestor of 
191 Petunioides then only maintained in N. attenuata. Together, these results suggest that tissue-

192 specific gain-of-expression in an existing gene, NaIFR3, have resulted in the evolution of the BA biosynthesis that mediates both pollinator attraction and florivore deterrence.

In summary, this study demonstrated that a new adaptive metabolic pathway in plants can arise from expression changes in a single gene. Such mechanism underlying the emergence of new metabolic pathways that mediate key ecological interactions might not only explain the evolution of amazing diversity of specialized metabolites in plants ${ }^{23,24}$, but also highlight the potential for breeding eco-friendly crops via metabolic engineering. 


\section{Methods}

201

Plant material. The N. attenuata Utah (UT) and Arizona (AZ) wild type seeds were originally collected from plants growing in a large natural population near Santa Clara, Utah, $\mathrm{USA}^{25}$, and a 20-plant population near Flagstaff, Arizona, USA ${ }^{26}$. They were inbred for 31 and 22 generations, respectively, in the glasshouse. Seeds of the G2 accession were collected in Utah as described in ${ }^{27}$. Additional natural accessions were collected by Ian T. Baldwin throughout the southwestern United States and inbred for one generation in the glasshouse ${ }^{28}$. To develop the AI-RIL population, UT and AZ were first crossed to generate F1 plants, which were then self-fertilized to generate $150 \mathrm{~F} 2$ plants. From F2 to F6, in each generation, we intercrossed $\sim 150$ progeny using a random mating and equal contribution crossing design ${ }^{29}$. For generation F7, two seeds from each of the crosses at F6 were germinated and used for the single-seed descendent inbreeding process. In total, five generations of inbreeding were conducted.

Plant growth. All seeds were germinated following the protocol described by Krügel et al. $(2002)^{30}$. Plants were grown under in glasshouse conditions $\left(26 \pm 1{ }^{\circ} \mathrm{C} ; 16 \mathrm{~h}: 8 \mathrm{~h} \text {, light: dark }\right)^{30}$. For the VIGS and transient transformation assay, plants were grown in a climate chamber under a constant temperature of $26^{\circ} \mathrm{C}$ and $16 \mathrm{~h}: 8 \mathrm{~h}$ (light: dark) light regime, and $65 \%$ relative humidity ${ }^{31}$.

Sampling of floral and foliar BA emissions. We measured floral BA from flowers of Nicotiana plants ( $\sim 50$ days after germination). Flowers of Nicotiana species remain open for three days and the flower age affects the quantity of floral volatiles ${ }^{32}$. Therefore, we removed all open flowers in the morning (7:00-9:00) of the day of volatile trapping. For each biological replicate, one freshly opened flower was taken out of the plant at 20:00 and incubated with polydimethylsiloxane (PDMS) tubes in a sealed $8 \mathrm{~mL}$ glass vial (MACHEREY-NAGEL) ${ }^{33}$.

To measure foliar BA emission, a transformed rosette leaf of $N$. attenuata was taken and incubated with PDMS tubes in a sealed $8 \mathrm{~mL}$ glass vial (MACHEREY-NAGEL).

Volatile analysis by TD-GC-MS. PDMS tubes were placed in an autosampler with thermal desorption unit (TDU; TD-20, Shimazu), which was connected to a quadruple GC-MS (QP2010-Ultra, Shimazu) for analysis. Specifications for desorption conditions, the used columns and the spectra reading and identification, were as described by Kallenbach et al. ${ }^{33}$ and Schuman et $a .^{34}$. For all of the volatiles, a 1:100 split was used to avoid overloading the detector.

Isotope-labeled phenylalanine feeding assay. Shoots with mature flower buds from UT plants (50 days after germination) were cut, inserted into a falcon tube $(50 \mathrm{~mL})$ and fed with either 10 mM L-phenyl-D5-alanine (Sigma-Aldrich, Cat\# 615870) or water for 24 hours, respectively.

Heterologous expression and enzyme assays of NaPAL1-4. The E. coli strain BL21 Star (DE3) (Thermo-Fisher) was used for expression of the complete open reading frames of 
NaPAL1-4. Since expression of native NaPAL4 yielded no protein, a codon optimized version was synthesized and used for enzyme characterization. Cultures were grown at $37^{\circ} \mathrm{C}$, induced at an $\mathrm{OD}_{600}=0.6$ with $1 \mathrm{mM}$ IPTG, subsequently placed at $18^{\circ} \mathrm{C}$, and grown for another 20 hours. The cells were collected by centrifugation and disrupted by freezing in liquid nitrogen and following thawing (five times) in chilled extraction buffer $(50 \mathrm{mM}$ Tris-HCl, $500 \mathrm{mM}$ $\mathrm{NaCl}, 20 \mathrm{mM}$ Imidazole, 10\% Glycerol; 1\% Tween20; pH8,6). Cell fragments were removed by centrifugation at $14,000 \mathrm{~g}$, the supernatant was purified with HisPur Cobalt Resin (Thermo-Fisher), and the purified proteins were concentrated with Amicon Ultra- 0.5 Centrifugal Filter devices (Merck Millipore) following manufactures instructions. To determine the catalytic activity of recombinant PAL enzymes, assays containing $20 \mu 1$ of the purified protein, $79 \mu \mathrm{l}$ assay buffer ( $50 \mathrm{mM}$ Tris- $\mathrm{HCl}, 500 \mathrm{mM} \mathrm{NaCl}, 10 \%$ Glycerol; pH8,6) and $1 \mathrm{mM}$ phenylalanine as substrate were incubated for 3 hours at $35^{\circ} \mathrm{C}$. Reaction products were analyzed using LC-MS/MS.

LC-MS/MS analysis of NaPAL enzyme products. Chromatography was performed on an Agilent 1260 Infinity II HPLC system (Agilent Technologies). Separation was achieved on a Zorbax Eclipse XDB-C18 column $(50 \times 4.6 \mathrm{~mm}, 1.8 \mu \mathrm{m}$, Agilent $)$. As mobile phases A and $\mathrm{B}$, formic acid $(0.05 \%)$ in water and acetonitrile were employed, respectively, with a mobile flow rate of $1.1 \mathrm{~mL} / \mathrm{min}$. The elution profile was: $0-0.5 \mathrm{~min}, 10 \% \mathrm{~B}$; $0.5-4.0 \mathrm{~min}, 10-90 \% \mathrm{~B}$; 4.0-4.02 $\min 90-100 \%$ B; 4.02-5.50 $\min 100 \% \mathrm{~B} ; 5.51-8.00 \min 10 \% \mathrm{~B}$. The column temperature was set at $20^{\circ} \mathrm{C}$. The liquid chromatography was coupled to an API-6500 tandem mass spectrometer (Sciex) equipped with a Turbospray ion source (ion spray voltage, -4500 $\mathrm{eV}$; turbo gas temperature, $650{ }^{\circ} \mathrm{C}$; nebulizing gas 60 psi., heating gas 60 psi, curtain gas 45 psi, collision gas medium). Measurements were performed in negative mode. Multiple reaction monitoring (MRM) was used to monitor a parent ion $\rightarrow$ product ion reaction for the PAL substrate phenylalanine $(\mathrm{m} / z 164 \rightarrow 147.0, \mathrm{CE}-18 \mathrm{~V}, \mathrm{DP}-50 \mathrm{~V})$ and the reaction product cinnamic acid $(\mathrm{m} / z 147 \rightarrow 103.0, \mathrm{CE}-16 \mathrm{~V}, \mathrm{DP}-50 \mathrm{~V})$. Analyst 1.6.3 software was used for data processing and analysis (AB Sciex).

Virus-induced gene silencing, VIGS. Leaves of young $N$. attenuata plants were agroinfiltrated with $p B I N T R A$ and $p T V-N a P A L 1 / 2 / 3 / 4$ or $p T V$-NaIFR3 according to a published protocol optimized for VIGS in N. attenuata ${ }^{35}$. Plants co-infiltrated with $p B I N T R A$ and $p T V 00$ were used as control. All VIGS experiments were repeated at least three times.

Southern blot analysis. A total amount of $20 \mu \mathrm{g}$ genomic DNA was digested overnight at $37^{\circ} \mathrm{C}$ with $100 \mathrm{U}$ EcoRV or XbaI or BamHI or HindIII (New England Biolabs) in independent reactions. The digested DNA was separated on a $0.8 \%(\mathrm{w} / \mathrm{v})$ agarose gel for $15 \mathrm{~h}$ at 30 Volt. DNA was blotted overnight onto a Gene Screen Plus Hybridization Transfer Membrane (Perkin-Elmer) using the capillary transfer method. For hybridizations, gene specific fragments that were used for VIGS (primer pairs listed in Table S1) were radiolabeled with [ $\alpha-32 \mathrm{P}] \mathrm{dCTP}$ (Perkin-Elmer) using the Rediprime II DNA Labeling System (GE Healthcare) according to the manufacturer's instructions. The blot was washed twice at high stringency $(0.1 \times$ SSC and $0.5 \%$ SDS for $20 \mathrm{~min})$. Membranes hybridized with radioactive probes were exposed for 12 hours to a phosphor screen (FUJIFILM imaging plate, BAS-IP MS 2340) in 
FUJIFILM BAS cassette 2340. Then the phosphor screen was scanned by Fujifilm FLA-3000 fluorescence laser imaging scanner for visualization.

\section{Transient transformation for subcellular localization analysis and ectopic expression in} leaves. The construction of 35S::YFP, 35S::YFP-NaPAL4, 35S::YFP-NaIFR3 and 35S::YFP$N a C H A L 3$ reporter fusions were carried out as described by Earley et al. ${ }^{36}$. The open reading frame encoding these genes were amplified and introduced into intermediate pENTR plasmid (Thermo-Fisher, Cat\# K240020) and then introduced into pEarleyGate 104 to generate YFP fusion constructs. The used primers are listed in Table S1. Recombined plasmids were then transformed into Agrobacterium tumefaciens strain GV3101 for subsequent plant transformation. Leaves of 3-weeks old $N$. attenuata plants were co-infiltrated with $A$. tumefaciens cells containing different plasmids. To detect the localization of NaPAL4, 35S::XA10-CFP was co-transformed with NaPAL4 and to generate endoplasmic reticulum (ER)-specific CFP fluorescent signal ${ }^{37,38}$. Fluorescence was visualized $48 \mathrm{~h}$ following the inoculation with a Zeiss LSM 510 Meta confocal microscope (Carl Zeiss).

Phylogenetic tree construction. The phylogenetic relationship among $22 N$. attenuata accessions was constructed using genome-wide SNP data. In brief, each accession was sequenced in low coverage (5-10 X) using Illumina HiSeq 2000 (pair-end). The short reads were mapped to $N$. attenuata reference genome ${ }^{21}$ using BWA-mem ${ }^{39}$. Genome-wide variants were called using GATK pipeline. VCFtools ${ }^{40}$ was used to remove non biallelic variants, reads coverage less than 1 or greater than 1000 , missing data in more than $40 \%$ of accessions, minimum SNP quality less than 30, mapping quality less than 50 and indels. BCFtools ${ }^{41}$ was used to prune SNPs in linkage (if two SNPs in a $1000 \mathrm{~kb}$ window have their $\mathrm{r}^{2}>0.5$ were discarded). This resulted in 157,833 high quality SNPs. These SNPs were used for building phylogenetic tree using RAxML-NG (v0.9.0) ${ }^{42}$ with 100 bootstraps. The best tree inference model "TVM+G4" was estimated by modeltest- $\mathrm{NG}^{43}$. To construct the phylogenetic tree of PAL4, IFR 3 and CHAL3, we used the Fishing Gene Family pipeline ${ }^{44}$ with minor modifications. In brief, the protein sequence of each of the three gene was used as the bait and genomic sequences of different Solanaceae species were used as the database. The extracted exon sequences were then aligned using GeneWise and the phylogenetic tree was constructed using PhyML (v3.3.3) ${ }^{45}$. The phylogenetic tree of seven Nicotiana species was constructed using PhyML(v3.3.3 $)^{45}$ based on partial nepGS gene sequences obtained from Clarkson et al. $2010^{46}$. Visualization of phylogenetic tree were conducted by iTOL v4.4.2 $2^{47,48}$.

QTL mapping. The genotype information of all AI-RIL plants and the linkage map were obtained from the dataset reported earlier ${ }^{13}$. The R package QTLRel was used for QTL mapping following the tutorial ${ }^{49}$. Briefly, the relationship among different individuals was first estimated based on pedigree information. The peak area of each compound was logtransformed. Samples with missing genotype or phenotype information were removed. In total, 207 samples were used for QTL mapping. Then the variance of the traits within the population was estimated via "estVC" and missing information of the genotypes was imputed using the function "genoImpute". The estimated trait variance and imputed genotypes were then used for the genome-wide scan. The empirical threshold was estimated based on 500 

fitting a multiple QTL model using the function "gls".

Quantitative RT-PCR. Total RNA was isolated using the RNeasy Plant Mini Kit (QIAGEN, Cat\#74903), and 1000 ng of total RNA were reverse transcribed using the PrimeScript RTqPCR Kit (TaKaRa, Cat\#RR037B). At least four independent biological replicates were collected and analyzed. RT-qPCR was performed on the Stratagene 500 MX3005P using a SYBR Green reaction mix (Eurogentec, Cat\#10-SN2X-03T). The primers used for mRNA detection of target genes by RT-qPCR are listed in Table S1. The mRNA of N. attenuata elongation factor $(\mathrm{NaEF})$ was used as internal control. three candidate genes among $22 \mathrm{~N}$. attenuata natural accessions, we firstly applied square root transformation followed by Z-Score normalization with "scale" function in R (https://cran.rproject.org). Then linear regression models were used to fit the transformed data. We used floral BA emission as response variable and transcript abundances of each gene as independent variable using the "lm" function in R.

Data availability. The data generated or analyzed during the current study are included in this published article (and its Supplementary Information) or are available from the corresponding author on reasonable request. 


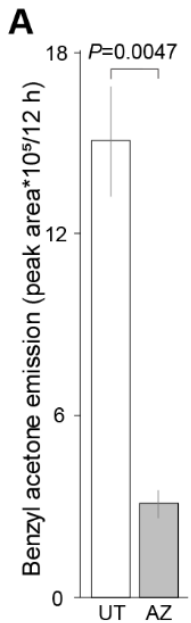

C

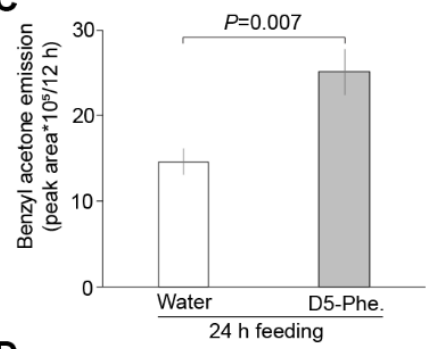

D



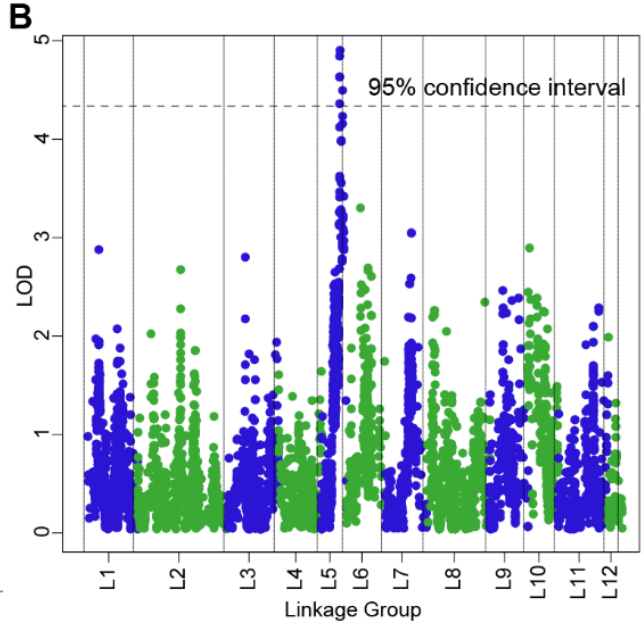

E

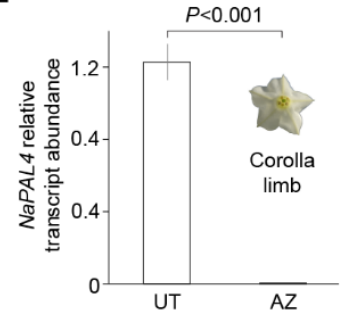

$\mathbf{F}$

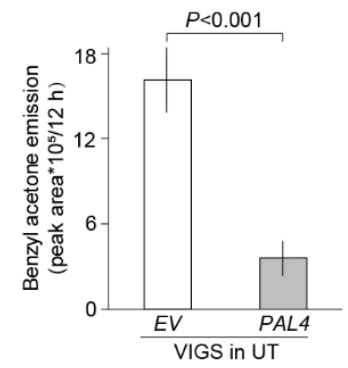

Figure 1. Phenylalanine ammonia-lyase 4 (NaPAL4) is involved in BA biosynthesis

(A) Quantitative differences in floral BA emission (mean $\pm \mathrm{SE}, \mathrm{n}=8$ ) between UT and AZ genotypes.

(B) Floral BA emission is mapped to one QTL locus. The QTL locus on linkage group 5 is marked. The $95 \%$ confidence interval is indicated with a dashed line. LOD, log of the odds. (C) Significant differences of both nocturnal floral BA emission (mean $\pm \mathrm{SE}, \mathrm{n}=8$ ) (top panel) and deuterium 5 -BA (D5-BA) (bottom panel) are shown between water and L-Phenyl$\mathrm{d}_{5}$-alanine (D5-Phe.) feeding on UT for 24 hours. Extracted-ion chromatogram, EIC.

(D) Transcript abundance of NaPAL4 (mean $\pm \mathrm{SE}, \mathrm{n}=4$ ) in corolla limb is different between UT and AZ genotypes. Corolla limb samples were harvested at 20:00. Transcript abundance was analyzed by RT-qPCR and relative to elongation factor gene in N. attenuata (NaEF).

(E) In comparison to VIGS-EV plants, the levels of nocturnal floral BA emission (mean $\pm \mathrm{SE}$, $\mathrm{n}=8$ ) of VIGS-NaPAL4 plants were significantly lower.

For (A), (C) and (E), the trapping of floral BA was performed for 12 hours from 20:00 to 8:00.

For (A), (C), (D) and (E), $P$ values were calculated using Student's- $t$ tests. 
A

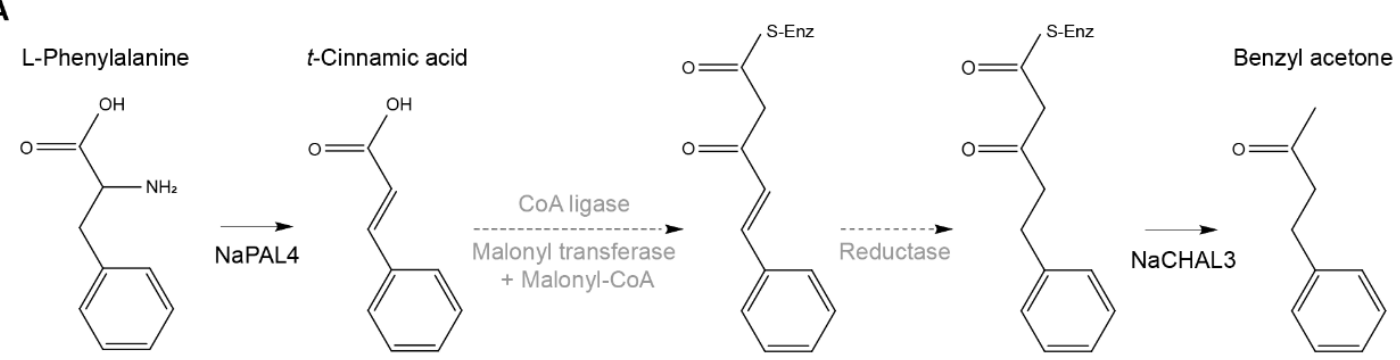

B



c

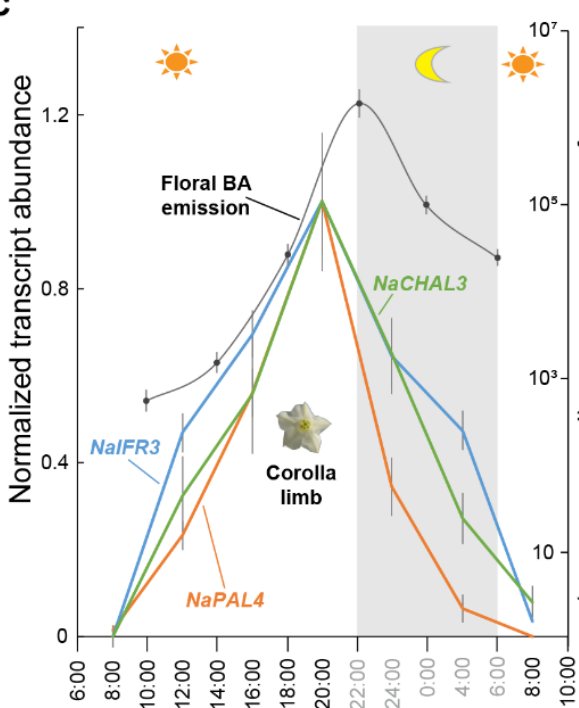

D

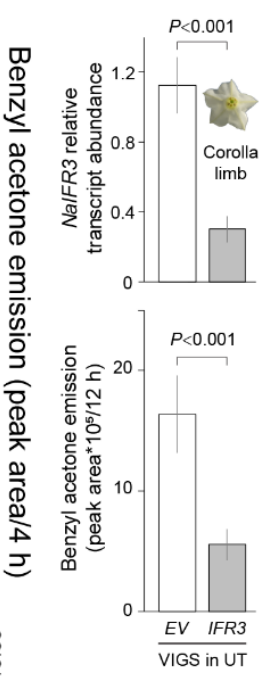

Figure 2. NaIFR3 is co-transcribed with NaPAL4 and NaCHAL3 and involved in BA biosynthesis

(A) Predicted biosynthesis pathway of BA. Dashed lines with arrow heads indicate the putative steps and the putative corresponding enzymes are shown in grey.

(B) NaPAL4, NaIFR3 and NaCHAL3 (mean $\pm \mathrm{SE}, \mathrm{n}=4$ ) are co-transcribed abundantly in corolla limb. Transcript abundance was analyzed by RT-qPCR. Different tissues were harvested at 20:00. The transcript abundance of each gene is relative to $N a E F$ and normalized (normalized as $\mathrm{X}^{\prime}=\mathrm{X} / \mathrm{X}_{\max }$ ) among different tissues.

(C) Emission kinetics of floral BA (mean $\pm \mathrm{SE}, \mathrm{n}=8$ ) are consistent to kinetics of NaPAL4, NaIFR 3 and NaCHAL3 transcription in corolla limb (mean $\pm \mathrm{SE}, \mathrm{n}=4$ ). The trapping of floral BA was performed for the periods of 4 hours, e.g. 8:00-12:00, 12:00-16:00 and so on. Corolla limb samples were harvested every 4 hours, e.g. 8:00, 12:00 and so on. Transcript abundance of each gene was analyzed by RT-qPCR and relative to $N a E F$ and normalized (normalized as $\mathrm{X}^{\prime}=\mathrm{X} / \mathrm{X}_{\max }$ ) among different time points.

(D) In comparison to VIGS- $E V$ plants, the levels of nocturnal floral BA emission (mean $\pm \mathrm{SE}$, $\mathrm{n}=8$ ) of VIGS-NaIFR3 plants were significantly lower. The trapping of floral BA was performed for 12 hours from 20:00 to 8:00. $P$ values were calculated using Student's- $t$ tests. 
A

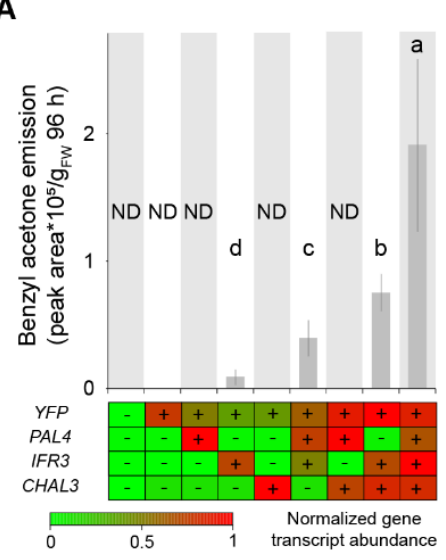

Figure 3. Ectopic expression of NaIFR3 is sufficient for foliar BA emission and transcriptional changes of $\mathrm{NaPAL4}, \mathrm{NaIFR} 3$ and $\mathrm{NaCHAL} 3$ contributed to the intraspecific variations of floral $B A$ emission

(A) Foliar BA emission (mean $\pm \mathrm{SE}, \mathrm{n}=8$ ) (top panel) with corresponding ectopic transcription of NaPAL4, NaIFR3 and NaCHAL3 (bottom panel) are shown. The trapping of foliar BA was performed for 96 hours after transformation. Different letters indicate significant differences in a Tukey-corrected post-hoc test following a one-way ANOVA $(P<$ 0.05). ND: Not detected. The complete open reading frames of NaPAL4, NaIFR3 and $\mathrm{NaCHAL} 3$ were fused downstream of YFP and transiently expressed in $N$. attenuata rosette leaves, individually or in different combinations together. Heatmap (bottom panel) representing the normalized transcript abundances of NaPAL4, NaIFR3 and NaCHAL3 (relative to $N a E F$ and normalized as $\mathrm{X}^{\prime}=\mathrm{X} / \mathrm{X}_{\max }$ among different transformations), respectively. + or -, presence or absence of the respective transgene.

(B) Heatmap representing the transcript abundances of NaPAL4, NaIFR3, NaCHAL3 and nocturnal floral BA emission in $22 \mathrm{~N}$. attenuata natural accessions. The phylogenetic tree of the 22 accessions on left panel was built based on their genomic sequences. Numbers on branches indicate the bootstrap percentage values calculated from 100 replicates, and only values greater than $50 \%$ are shown. The transcript abundance is relative to $N a E F$ and normalized as $\mathrm{X}^{\prime}=\mathrm{X} / \mathrm{X}_{\max }$ among different accessions. The trapping of floral BA was performed for the 12 hours from 20:00 to 8:00.

(C) Relationship between floral BA emission and transcript abundance of NaPAL4, NaIFR3 and NaCHAL3 in 22 natural accessions. The y-axis denotes the proportion of BA variation that could be explained by the changes of genes' transcription. 
A

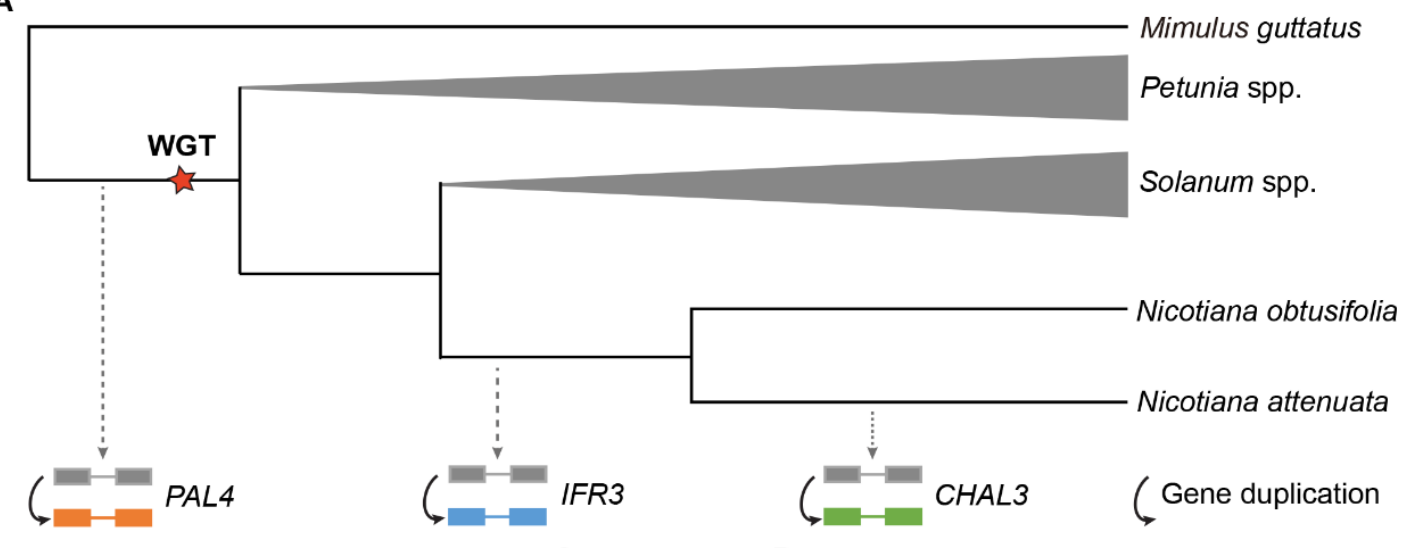

B

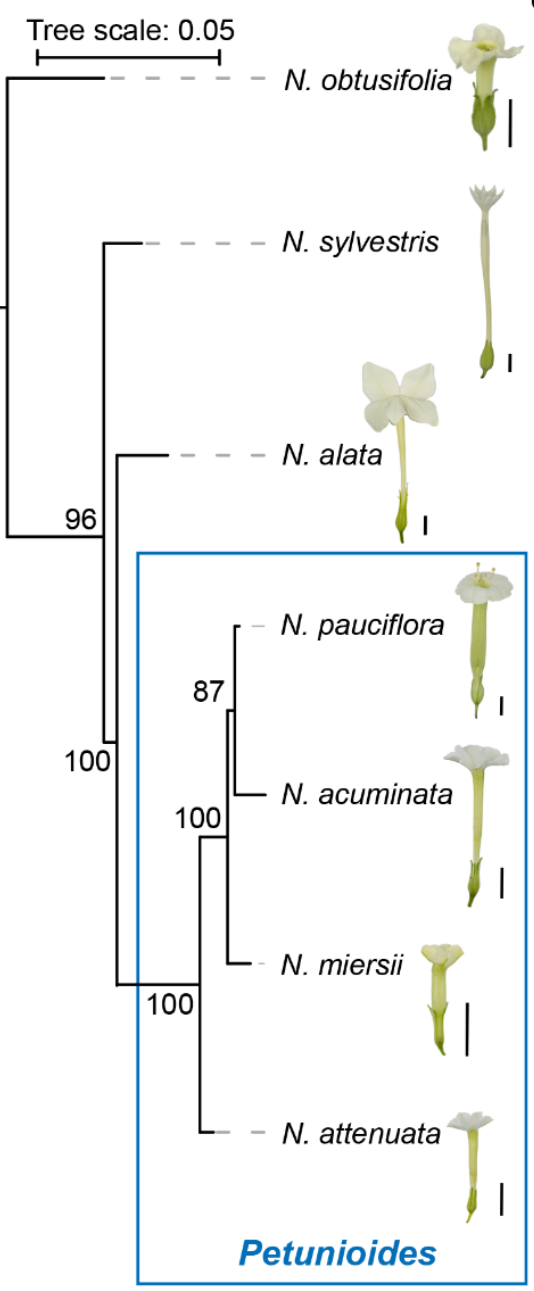

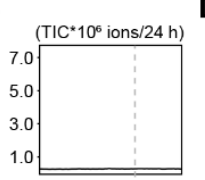
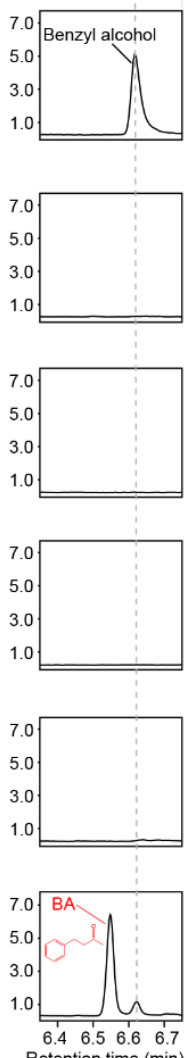

Retention time 6.7

D

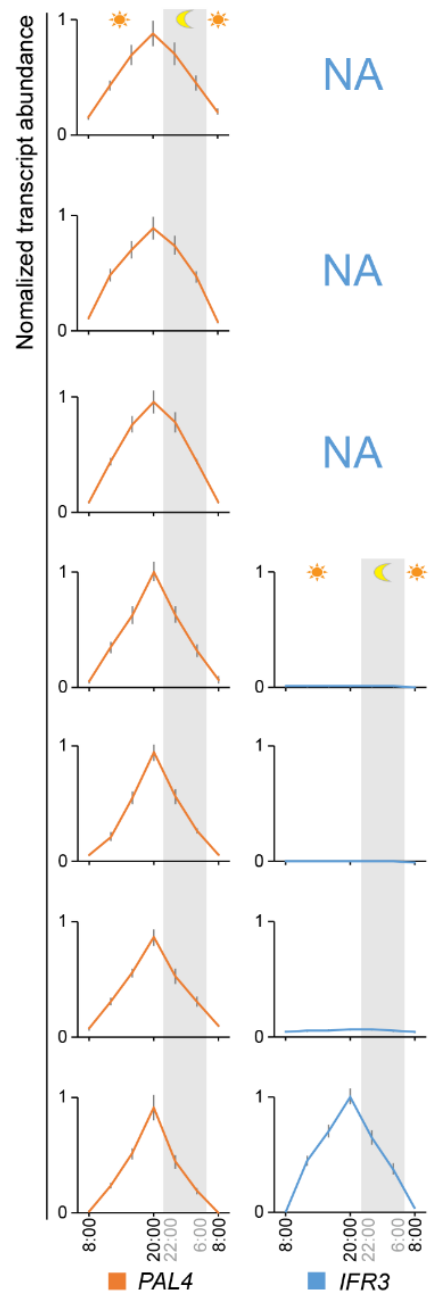

NA

NA

NA
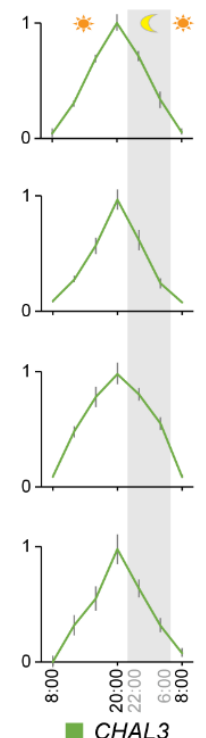

Figure 4. Gain of corolla limb expression of NaIFR3 coincides with floral BA

(A) Schematic evolutionary history of NaPAL4, NaIFR3 and NaCHAL3. WGT, whole genome triplication. Dashed lines with arrow heads indicate the estimated time points of corresponding gene duplication.

(B) The phylogenetic tree of the seven Nicotiana species was built based on partial nepGS sequences. Numbers on branches indicate the bootstrap percentage values calculated from 1000 replicates, and only values greater than $50 \%$ are shown. Scale bars of flowers: $1 \mathrm{~cm}$. 
421 (C) Floral emission of BA is species-specific in $N$. attenuata. The trapping of floral volatile was started at 8:00 and was performed for 24 hours. Total ion current, TIC. (D) Kinetic of the transcript abundance of NaPAL4 (left panel), NaIFR3 (middle panel) and NaCHAL3 (right panel) in corolla limbs among seven Nicotiana species. Transcript abundance (mean $\pm \mathrm{SE}, \mathrm{n}=4$ ) was analyzed by conserved primers and RT-qPCR. The transcript abundance of each gene is relative to $N a E F$ and normalized (normalized as $\mathrm{X}^{\prime}=$ $\left.\mathrm{X} / \mathrm{X}_{\max }\right)$ among different species in kinetics. When the gene could not be identified from either genomic data or homologous cloning, we labeled the expression as NA (not available). 
429

430

431

432

433

434

435

436

437

438

439

440

441

442

443

444

445

446

447

448

449

450

451

452

453

454

455

456

457

458

459

460

461

462

463

464

465

466

467

468

469

470

471

472

\section{References:}

1 Raguso, R. A. Wake up and smell the roses: the ecology and evolution of floral scent. Annu. Rev. Ecol. Syst. 39, 549-569 (2008).

2 Muhlemann, J. K., Klempien, A. \& Dudareva, N. Floral volatiles: from biosynthesis to function. Plant Cell Environ. 37, 1936-1949 (2014).

3 Raguso, R. A. More lessons from linalool: insights gained from a ubiquitous floral volatile. Curr. Opin. Plant Biol. 32, 31-36 (2016).

4 Schiestl, F. P. Ecology and evolution of floral volatile-mediated information transfer in plants. New Phytol. 206, 571-577 (2015).

5 Wong, D. C. J., Pichersky, E. \& Peakall, R. The biosynthesis of unusual floral volatiles and blends involved in orchid pollination by deception: current progress and future prospects. Front. Plant Sci. 8 (2017).

6 Euler, M. \& Baldwin, I. T. The chemistry of defense and apparency in the corollas of Nicotiana attenuata. Oecologia 107, 102-112 (1996).

7 Baldwin, I. T., Preston, C., Euler, M. \& Gorham, D. Patterns and consequences of benzyl acetone floral emissions from Nicotiana attenuata plants. J. Chem. Ecol. 23, 2327-2343 (1997).

8 Kessler, D., Gase, K. \& Baldwin, I. T. Field experiments with transformed plants reveal the sense of floral scents. Science 321, 1200-1202 (2008).

9 Kessler, D. et al. How scent and nectar influence floral antagonists and mutualists. Elife 4 (2015).

10 Haverkamp, A. et al. Hawkmoths evaluate scenting flowers with the tip of their proboscis. Elife 5 (2016).

11 Kessler, D., Bing, J., Haverkamp, A. \& Baldwin, I. T. The defensive function of a pollinator-attracting floral volatile. Funct. Ecol. 33, 1223-1232 (2019).

12 Haverkamp, A., Bing, J., Badeke, E., Hansson, B. S. \& Knaden, M. Innate olfactory preferences for flowers matching proboscis length ensure optimal energy gain in a hawkmoth. Nat. Commun. 7, 11644 (2016).

13 Zhou, W. et al. Tissue-specific emission of (E)-alpha-bergamotene helps resolve the dilemma when pollinators are also herbivores. Curr. Biol. 27, 1336-1341 (2017).

14 Widhalm, J. R. \& Dudareva, N. A familiar ring to it: biosynthesis of plant benzoic acids. Mol. Plant 8, 83-97 (2014).

15 Achnine, L., Blancaflor, E. B., Rasmussen, S. \& Dixon, R. A. Colocalization of Lphenylalanine ammonia-lyase and cinnamate 4-hydroxylase for metabolic channeling in phenylpropanoid biosynthesis. Plant Cell 16, 3098-3109 (2004).

16 Brockmöller, T. et al. Nicotiana attenuata Data Hub (NaDH): an integrative platform for exploring genomic, transcriptomic and metabolomic data in wild tobacco. $B M C$ Genomics 18, 79 (2017).

17 Koeduka, T. et al. Eugenol and isoeugenol, characteristic aromatic constituents of spices, are biosynthesized via reduction of a coniferyl alcohol ester. Proc. Natl Acad. Sci. U S A 103, 10128-10133 (2006).

18 Haverkamp, A., Hansson, B. S., Baldwin, I. T., Knaden, M. \& Yon, F. Floral trait variations among wild tobacco populations influence the foraging behavior of hawkmoth pollinators. Front. Ecol. Evol. 6 (2018). 
Abe, I., Sano, Y., Takahashi, Y. \& Noguchi, H. Site-directed mutagenesis of benzalacetone synthase. The role of the $\mathrm{Phe}^{215}$ in plant type III polyketide synthases. $J$. Biol. Chem. 278, 25218-25226 (2003).

20 Abe, I., Takahashi, Y., Morita, H. \& Noguchi, H. Benzalacetone synthase. A novel polyketide synthase that plays a crucial role in the biosynthesis of phenylbutanones in Rheum palmatum. Eur. J. Biochem. 268, 3354-3359 (2001).

$21 \mathrm{Xu}, \mathrm{S}$. et al. Wild tobacco genomes reveal the evolution of nicotine biosynthesis. Proc. Natl Acad. Sci. US A 114, 6133-6138 (2017).

22 Clarkson, J. J., Dodsworth, S. \& Chase, M. W. Time-calibrated phylogenetic trees establish a lag between polyploidisation and diversification in Nicotiana (Solanaceae). Plant Syst. Evol. 303, 1001-1012 (2017).

23 Fang, C., Fernie, A. R. \& Luo, J. Exploring the diversity of plant metabolism. Trends in Plant Science 24, 83-98, doi:https://doi.org/10.1016/j.tplants.2018.09.006 (2019).

24 Pichersky, E. \& Lewinsohn, E. Convergent evolution in plant specialized metabolism. Annu. Rev. of Plant Biol. 62, 549-566, doi:10.1146/annurev-arplant-042110-103814 (2011).

25 Halitschke, R., Kessler, A., Kahl, J., Lorenz, A. \& Baldwin, I. T. Ecophysiological comparison of direct and indirect defenses in Nicotiana attenuata. Oecologia 124, 408417 (2000).

26 Glawe, G. A., Zavala, J. A., Kessler, A., van Dam, N. M. \& Baldwin, I. T. Ecological costs and benefits correlated with trypsin protease inhibitor production in Nicotiana attenuata. Ecology 84, 79-90 (2003).

27 Schuman, M. C., Heinzel, N., Gaquerel, E., Svatos, A. \& Baldwin, I. T. Polymorphism in jasmonate signaling partially accounts for the variety of volatiles produced by Nicotiana attenuata plants in a native population. New Phytol. 183, 1134-1148 (2009).

28 Li, D., Baldwin, I. T. \& Gaquerel, E. Navigating natural variation in herbivory-induced secondary metabolism in coyote tobacco populations using MS/MS structural analysis. Proc. Natl Acad. Sci. US A 112, E4147-4155 (2015).

29 Rockman, M. V. \& Kruglyak, L. Breeding designs for recombinant inbred advanced intercross lines. Genetics 179, 1069-1078 (2008).

30 Krügel, T., Lim, M., Gase, K., Halitschke, R. \& Baldwin, I. T. Agrobacterium-mediated transformation of Nicotiana attenuata, a model ecological expression system. Chemoecology 12, 177-183 (2002).

31 Galis, I. et al. The use of VIGS technology to study plant-herbivore interactions. Methods Mol. Biol. 975, 109-137 (2013).

32 Bhattacharya, S. \& Baldwin, I. T. The post-pollination ethylene burst and the continuation of floral advertisement are harbingers of non-random mate selection in Nicotiana attenuata. Plant J. 71, 587-601 (2012).

33 Kallenbach, M. et al. A robust, simple, high-throughput technique for time-resolved plant volatile analysis in field experiments. Plant J. 78, 1060-1072 (2014).

34 Schuman, M. C., Barthel, K. \& Baldwin, I. T. Herbivory-induced volatiles function as defenses increasing fitness of the native plant Nicotiana attenuata in nature. Elife $\mathbf{1}$ (2012).

35 Saedler, R. \& Baldwin, I. T. Virus-induced gene silencing of jasmonate-induced direct 
defences, nicotine and trypsin proteinase-inhibitors in Nicotiana attenuata. J. Exp. Bot. 55, 151-157 (2004).

Earley, K. W. et al. Gateway-compatible vectors for plant functional genomics and proteomics. Plant J. 45, 616-629 (2006). death and calcium depletion in the endoplasmic reticulum. Plant Cell 26, 497-515 (2014).

Li, R. et al. A terpenoid phytoalexin plays a role in basal defense of Nicotiana benthamiana against Potato virus X. Sci. Rep. 5, 9682 (2015).

$41 \mathrm{Li}, \mathrm{H}$. A statistical framework for SNP calling, mutation discovery, association mapping and population genetical parameter estimation from sequencing data. Bioinformatics 27, 2987-2993, doi:10.1093/bioinformatics/btr509 (2011).

Li, H. \& Durbin, R. Fast and accurate short read alignment with Burrows-Wheeler transform. Bioinformatics 25, 1754-1760 (2009).

Danecek, P. et al. The variant call format and VCFtools. Bioinformatics 27, 2156-2158 (2011). Kozlov, A. M., Darriba, D., Flouri, T., Morel, B. \& Stamatakis, A. RAxML-NG: a fast, scalable and user-friendly tool for maximum likelihood phylogenetic inference. Bioinformatics, doi:10.1093/bioinformatics/btz305 (2019).

Darriba, D. et al. ModelTest-NG: a new and scalable tool for the selection of DNA and protein evolutionary models. bioRxiv, 612903, doi:10.1101/612903 (2019). Zheng, H. et al. FGF: a web tool for Fishing Gene Family in a whole genome database. Nucleic. Acids Res. 35, W121-125 (2007).

Guindon, S., Delsuc, F., Dufayard, J. F. \& Gascuel, O. Estimating maximum likelihood phylogenies with PhyML. Methods Mol. Biol. 537, 113-137 (2009). synthetase evolution in Nicotiana: phylogenetics and the origins of allotetraploid and homoploid (diploid) hybrids. Mol. Phylogenet. Evol. 55, 99-112 (2010). Ciccarelli, F. D. et al. Toward automatic reconstruction of a highly resolved tree of life. Science 311, 1283-1287 (2006).

Letunic, I. \& Bork, P. Interactive Tree Of Life (iTOL) v4: recent updates and new developments. Nucleic. Acids Res. 47, W256-W259 (2019).

49 Cheng, R., Abney, M., Palmer, A. A. \& Skol, A. D. QTLRel: an R package for genomewide association studies in which relatedness is a concern. BMC Genet. 12, 66 (2011).

\section{Acknowledgments}

We thank the glasshouse team of the Max Planck Institute for Chemical Ecology for plant cultivation; Dapeng Li, Wenwu Zhou, Klaus Gase and Eva Rothe for technical assistance; Lei Hou, Rayko Halitschke, Danny Kessler and Martin Schäfer for fruitful discussions. This work was supported by funding from the Swiss National Science Foundation (PEBZP3-142886 to

558 S.X.), the Max Planck Society, and the Advanced Grant no. 293926 of the European Research 559 Council to I.T.B. 


\section{Author contributions}

562 Conceptualization, S.X and H.G; QTL mapping and gene candidate validation, S.X. and H.G.;

563 intra- and interspecific gene expression analysis, VIGS, isotope-labeled phenylalanine feeding

564 and Southern blot assay, H.G.; transient transformation for subcellular localization analysis

565 and ectopic expression in leaves, H.G. and R.L.; heterologous expression and enzyme assays

566 of NaPAL1-4, H.G., N.D.L. and T.G.K.; sampling of floral and foliar BA emissions and

567 volatile analysis by TD-GC-MS, H.G. and J.B.; phylogenetic analysis, S.X., H.G. and Y. W.;

568 writing - original draft, S.X. and H.G.; funding acquisition, S.X. and I.T.B.; resources, S.X.

569 and I.T.B.; supervision, S.X.

\section{Declaration of interests}

572 The authors declare that there is no conflict of interest regarding the publication of this article.

\section{Additional information}

575 Correspondence and requests for resources and reagents should be addressed to S.X.

576 Requesting N. attenuata seeds should be addressed to I.T.B. 